\title{
Learning Semantic-Level Information Extraction Rules by Type-Oriented ILP
}

\author{
Yutaka Sasaki and Yoshihiro Matsuo \\ NTT Communication Science Laboratories \\ 2-4 Hikaridai, Seika-cho, Soraku-gun, Kyoto 619-0237, Japan \\ \{sasalki, yosihiro\}@cslab.kecl.ntt.co.jp
}

\begin{abstract}
This paper describes an approach to using semantic representations for learning information extraction (IE) rules by a type-oriented inductive logic programming (ILP) system. NLP components of a machine translation system are used to automatically generate semantic representations of text corpus that can be given directly to an ILP system. The latest experimental results show high. precision and recall of the learned rules.
\end{abstract}

\section{Introduction}

Information extraction (IE) tasks in this paper involve the MUC-3 style IE. The input for the information extraction task is an empty template and a set of natural language texts that describe a restricted target domain, such as corporate mergers or terrorist attacks in South America. Templates have a record-like data structure with slots that have names, e.g., "company name" and "merger date", and values. The output is a set of filled templates. IE tasks are highly domain-dependent, so rules and dictionaries for filling values in the template slots depend on the domain.

It is a heavy burden for IE system developers that such systems depend on hand-made rules, which cannot be casily constructed and changed. For example, Umass/MUC-3 needed about 1,500 person-hours of highly skilled labor to build the IE rules and represent them as a dictionary (Lehnert, 1992). All the rules must be reconstructed from scratch when the target domain is changed.

To cope with this problem, some pioneers have studied methods for learning information extraction rules (Riloff,1996; Soderland et al., 1995; Kim el al., 1995; Huffman, 1996; Califf and Mooney, 1997). Along these lines, our ap- proach is to apply an inductive logic programming (IILP) (Muggleton, 1.991) system to the learning of IE rules, where information is extracted from semantic representations of news articles. The ILP system that wo employed is a type-oriented ILP system RIII+ (Sasalki and Haruno, 1997), which can efficiently and effectively handle type (or sort) information in training data.

\section{Our Approach to IE Tasks}

This section describes our approach to IF tasks. Figure 1 is an overvicw of our approach to learning IE rules using an ILP system from semantic representations. First, training articles are analyzed and converted into semantic representalions, which are filled case frames represented as atomic formulae. Training templates are prepared by hand as well. The ILP system learns II rules in the form of logic programs with type information. To extract key information from a new article, semantic representations automatically generated from the article is matched by the IIE rules. Extracted information is filled into the template slots.

\section{NLP Resources and Tools}

\subsection{The Semantic Attribute System}

We used the semantic attribute system of "GoiTaikei - A Japanese Lexicon" (Ikehara et al., 1997a; Kurohashi and Sakai, 1999) compiled by the NT.T Communication Science Laboratories for a Japanese-to-English machine translation system, AJT-J/E (Ikehara et al., 1994). The semantic attribute system is a sort of hierarchical concept thesaurus represented as a tree structure in which each node is called a semantic category. An edge in the tree represents an is_a or has_a relation between two categories. The semantic attribute system is 1.2 levels deep and 


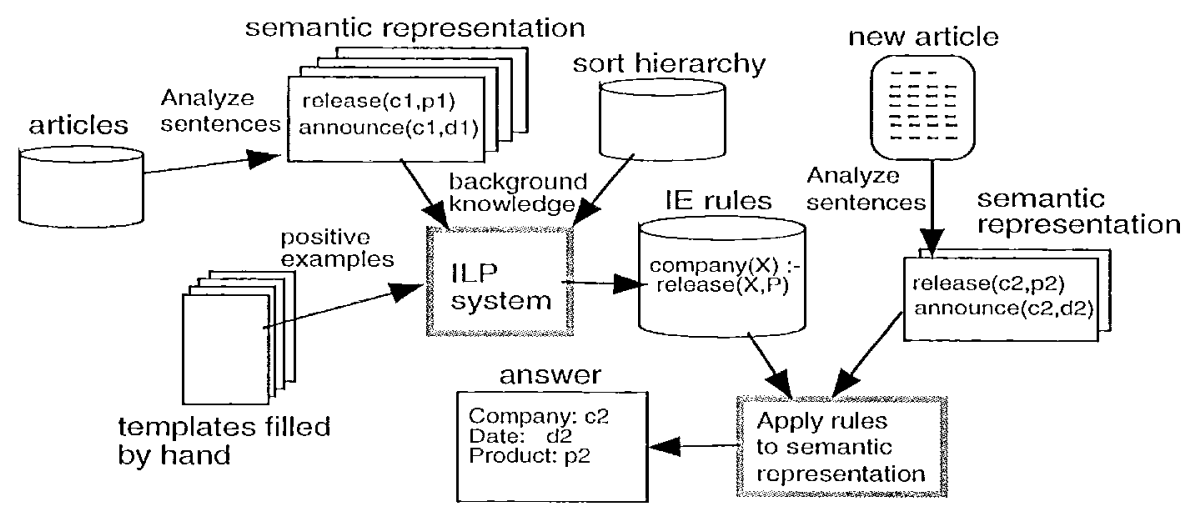

l'igure 1: Block diagram of Il', using II.P

contains about 3,000 semantic category nodes. More than 300,000 Japaneso words are linked to the category nodes.

\subsection{Verb Case Frame Dictionary}

The Japanese-to-Finglish valency pattern dictionary of "Goi-Taikci" (Ikehara et al., 1997b; Kurohashi and Sakai, 1999) was also originally developed for $\Lambda \mathrm{L} T-\mathrm{J} / \mathrm{E}$. The valency dictionary contains about 15,000 case frames with semantic restrictions on their arguments for 6,000 Japanese verbs. Wach case frame consists of one predicate and one or more case elements that have a list of semantic categories.

\subsection{Natural Language Processing Tools}

We used the NIT components of $\Lambda \mathrm{L}^{\prime} \mathrm{T}^{2} \mathrm{~J} / \mathrm{l}$ for text analysis. T'hese include the morphological analyzer, the syntactic analyzer, and the case analyzer for Japanese. The components are robust and generic tools, mainly targeted to nowspaper articles.

\subsubsection{Generic Case Analyzer}

Iet us examine the case analysis in more detail. 'The case analyzer reads a set of parse tree candidates produced by the Japanese syntactic analyzer. The parse tree is represented as a dependency of phrases (i.e., Japanese bunsetsu).

First, it divides the parse tree into unit sentences, where a unit sentence consists of one predicate and its noun and adverb dependent phrases. Second, it compares each unit semtence with a verb case frame dictionary. Wach frame consists a predicate condition and several case elements conditions. The predicate condition specifies a verb that matches the frame and each case-role has a case element condition which specifies particles and semantic catcgorjes of noun phrases. The preference value is defined as the summation of noun phrase preferences which are calculated from the distances between the categories of the input sentences and the categories written in the frames. The case analyzer then chooses the most preferable parse tree and the most preferable combination of case frames.

T'he valency dictionary also has casc-roles (T'able 1) for noun phrase conditions. The caseroles of adjuncts are determined by using the particles of adjuncts and the semantic catcgories of noun phrases.

As a result, the output of the case analysis is a set of case frames for each unit sentence. The noun phrases in frames are labeled by case-roles in Table 1.

lor simplicity, wo use case-role codes, such as N1. and N2, as the labels (or slot namos) to represent case frames. The relation between sentences and case-roles is described in detail in (Ikchara et al., 1993).

\subsubsection{Logical Form Translator}

Wo developed a logical form translator File that generates semantic representations $\mathrm{ex}$ pressed as atomic formulae from the case frames and parse trees. For later use, document ID and tense information are also added to the case frames.

lor example, the case frame in 'Table 2 is obtained after analyzing the following sentence of document D1:

"Jakku(Jack) ha sutsukesu(suitcase) wo 
Table 1: Case-Roles

\begin{tabular}{llll}
\hline Name & Code & Description & Example \\
\hline Subject & N1 & $\begin{array}{l}\text { the agent/experiencer of } \\
\text { an event/situation }\end{array}$ & I throw a ball. \\
Object1 & N2 & $\begin{array}{l}\text { the object of an event } \\
\text { another object of an event }\end{array}$ & $\begin{array}{l}\text { I throw a ball. } \\
\text { I compare it with them. }\end{array}$ \\
Object2 & N3 & $\begin{array}{l}\text { source location of a movement } \\
\text { Loc-Source }\end{array}$ & I start from Japan. \\
Loc-Goal & N5 & goal location of a movement & I go to Japan. \\
Purpose & N6 & the purpose of an action & I go shopping. \\
Result & N7 & the result of an event & It results in failure. \\
Locative & N8 & the location of an event & It occurs at the station. \\
Comitative & N9 & co-experiencer & I share a room with him. \\
Quotative & N10 & quoted expression & I say that .... \\
Material & N11 & matcrial/ingredient & I fill the glass with water. \\
Cause & N12 & the reason for an event & It collapsed from the weight. \\
Instrument & N13 & a concrete instrument & I speak with a microphone. \\
Means & N14 & an abstract instrument & I speak in Japanese. \\
Time-Position & TN1 & the time of an event & I go to bed at 10:00. \\
Time-Source & TN2 & the starting time of an. event & I work from Monday. \\
Time-Goal & TN3 & the end time of an event & It continues until Monday. \\
Amount & QUANT quantity of something & I spcnd \$10. \\
\hline
\end{tabular}

shokuba(the office) kara(from) kuko(the airport) ni(to) hakobu(carry)"

("Jack carries a suitcase from the office to the airport.")

Table 2: Case Frame of the Sample Sentence

\begin{aligned} & \hline predicate: hakobu (carry) \\ & article: D1. \\ & tense: present \\ & N1: Jakku (Jack) \\ & N2: sutsukesu (suitcase) \\ & N4: shokuba (the office) \\ & N5: kuko (the airport) \\ & \hline\end{aligned}

\section{Inductive Learning Tool}

Conventional ILP systems take a set of positive and negative examples, and background knowledge. The output is a set of lypotheses in the form of logic programs that covers positives and do not cover negatives. We employed the typeoriented ILP system RHB ${ }^{+}$.

\subsection{Features of Type-oriented ILP System RHB ${ }^{+}$}

The type-oriented II.P system has the following features that match the needs for learning IT: rules.

- A type-oriented ILP system can cfliciently and effectively handle type (or semantic category) information in training data. This feature is advantageous in controlling the generality and accuracy of learned IIE rules.

- It can directly use semantic representations of the text as background knowledge.

- It can learn from only positive examples.

- Predicates are allowed to have labels (or keywords) for readability and expressibility.

\subsection{Summary of Type-oriented ILP System RHB ${ }^{+}$}

This section summarizes the employed typeoriented ILP system $\mathrm{RHB}^{+}$. The input of $\mathrm{RHB}^{+}$is a set of positive examples and background knowledge including type hierarchy (or 
the semantic attribute system). The output is a set of IIorn clauses (I loyd, 1987) having variables with type information. 'That is, the term is extended to the $\tau$-term.

\section{3 $\tau$-terms}

$\tau$-terms are the restricted form of $\psi$-terms ( $\Lambda$ ïtKaci and Nasr, 1986; Aït-Kaci et al., 1994). Informally, $\tau$-terms are Prolog terms whose variables are replaced with variable $V a r$ of type $T$, which is denoted as Var:T. Predicate and function symbols are allowed to have features (or labcls). For example,

speak(agent $\Rightarrow X$ :human,object $\Rightarrow Y$ :language $)$ is a clause based on $\tau$-terms which has labels agent and object, and types human and language.

\subsection{Algorithm}

The algorithm of RHI3+ is basically a greedy covering algorithm. It constructs clauses oncby-one by calling inner_loop (Algorithm 1) which returns a hypothesis clause. A hypothesis clause is represented in the form of head:body. Covered examples are removed from $l$ ' in cach cycle.

The inner loop consists of two phases: the head construction phase and the body construction phase. It constructs heads in a bottom-up manner and constructs the body in a top-down manner, following the result described in (\%elle et. al., 1991).

The search heuristic $P W I$ is weighted informativity employing the Japlace estimate. Let $T=\{$ II ead $:-$ Body $\} \cup B K$.

$$
P W I(P, T)=-\frac{1}{|\hat{P}|} \times \log _{2} \frac{|\hat{P}|+1}{|Q(T)|+2},
$$

where $|\hat{P}|$ denotes the number of positive examples covered by $T$ and $Q\left(T^{\prime}\right)$ is the empirical content. 'The smaller the value of PWI, the candidate clause is better. $Q(T)$ is defined as the set of atoms (1) that are derivable from 'T and (2) whose predicate is the target predicate, i.e., the predicate name of the head.

The dynamic type restriction by posilive examples uses positive examples currently covered in order to determine appropriate types to variables for the current clause.

\section{Algorithm 1 inner_loop}

1. Given positives $P$, original positives $P_{0}^{\prime}$, background knowledge BK.

2. Decide types of variables in a head by computing the typed least general generalizations (lgg) of $N$ pairs of elements in $P$, and select the most gencral head as Head.

3. If the stopping condition is satisfied, return Head.

1. Let Body be empty.

5. Create a set of all possible literals $L$ using variables in Head and Body.

6. Set IBEAM be top $K$ literals $l_{k}$ of $L$ with. respect to the positive weighted informativity I'WI.

7. Do later steps, assuming that $l_{k}$ is added to Body for each literal $l_{k}$ in BEAM.

8. Dynamically restrict types in Body by calling the dynamic type restriction by positive cxamples.

9. If the stopping condition is satisficd, return (Ilead:- Body).

10. Goto 5 .

\section{Illustration of a Learning Process}

Now, we examine the two short notices of new products release in Table 3 . The following table shows a sample template for articles reporting a new product release.

\begin{tabular}{l} 
Template \\
1. article id: \\
2. company: \\
3. product: \\
4. release date: \\
\hline
\end{tabular}

\subsection{Preparation}

Suppose that the following semantic representations is obtained from Article 1 .

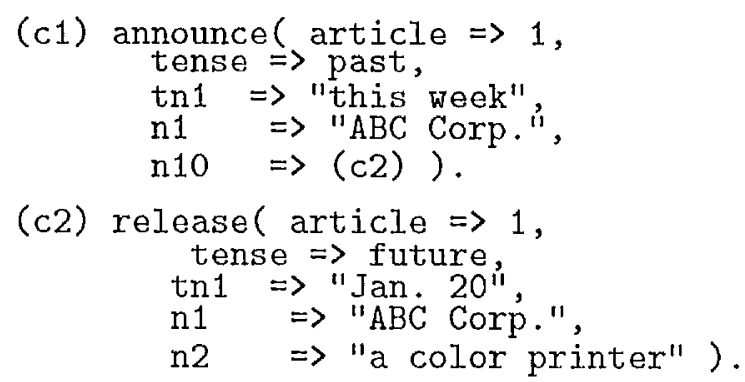


Table 3: Sample Sontences

\begin{tabular}{|c|c|}
\hline Article id & Sentence \\
\hline \hline$\# 1$ & "ABC Corp. this week announced that it will release a color printer on Jan. 20." \\
\hline$\# 2$ & "XYZ Corp. released a color scanncr last month." \\
\hline
\end{tabular}

The filled template for Article 1 is as follows.

\begin{tabular}{l} 
Template 1 \\
\hline 1. article id: 1 \\
2. company: $\Lambda \mathrm{BC}$ Corp. \\
3. product: a color printer \\
4. relcase date: Jan. 20 \\
\hline
\end{tabular}

Suppose that the following semantic representation is obtained from Article 2.

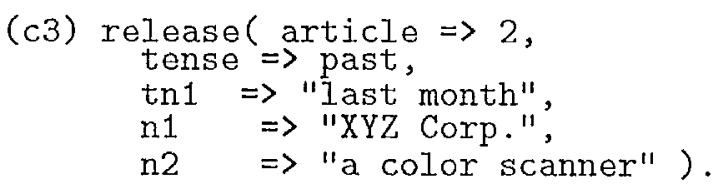

The filled template for Article 2 is as follows.

\section{T'omplate 2}

1. article id: 2

2. company: XYZ Corp.

3. product: a color scanner

4. release date: last month

\subsection{Head Construction}

'T'wo positive examples are selected for the template slot "company".

$$
\begin{gathered}
\text { company (article-number } \Rightarrow 1 \\
\text { name } \Rightarrow \text { "ABC Corp"). } \\
\text { company (article-number } \Rightarrow 2 \\
\text { name } \Rightarrow \text { "XYZ Corp"). }
\end{gathered}
$$

By computing a least general generalization (lgg)sasaki97, the following head is obtained:

$$
\begin{array}{r}
\text { company ( article-number } \Rightarrow \text { Art: number } \\
\text { name } \Rightarrow \text { Co: organization). }
\end{array}
$$

\subsection{Body Construction}

Generate possible literals ${ }^{1}$ by combining predicate names and variables, then check the PWI

\footnotetext{
1 "literals" here means atomic formulae or negated ones.
}

values of clauses to which one of the literal added. In this case, suppose that adding the following literal with predicate release is the best one. After the dynamic type restriction, the current clause satisfies the stopping condition. Finally, the rule for extracting "company name" is returned. Extraction rules for other slots "product" and "release date" can be learned in the same manner. Note that several literals may be needed in the body of the clause to satisfy the stopping condition.

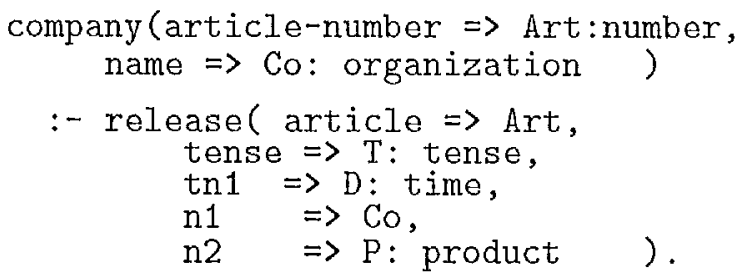

\subsection{Extraction}

Now, we have the following semantic representation extracted from the new article:

Article 3: "JPN Corp. has released a now CD) playcr." 2

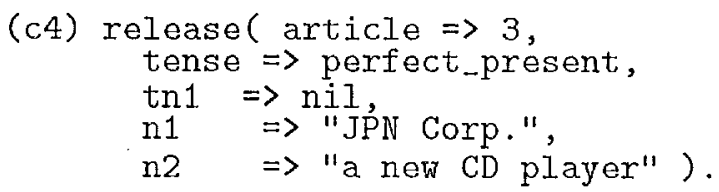

Applying the learned IE rules and other rules, we can obtain the filled template for $\Lambda$ rticle 3.

\begin{tabular}{l}
\hline Template 3 \\
\hline 1. article id: 3 \\
2. company: JPN Corp. \\
3. product: CD player \\
4. release date: \\
\hline
\end{tabular}

\footnotetext{
${ }^{2}$ We always assume nil for the case that is not included in the sentence.
} 
'Jable 4: Jearning results of new product release

(a) Without data correction

\begin{tabular}{|c|c|c|c|c|c|}
\hline & company & product & release date & announce date & price \\
\hline \hline Precision & $89.6 \%$ & $80.5 \%$ & $90.6 \%$ & $100.0 \%$ & $58.4 \%$ \\
Recall & $82.1 \%$ & $66.7 \%$ & $66.7 \%$ & $82.4 \%$ & $60.8 \%$ \\
Average time (sec.) & 15.8 & 22.1 & 14.1 & 2.2 & 11.0 \\
\hline
\end{tabular}

(b) With data correction

\begin{tabular}{|c|c|c|c|c|c|}
\hline & company & product & rolease date & announce date & price \\
\hline \hline Precision & $91.1 \%$ & $80.0 \%$ & $92.3 \%$ & $100.0 \%$ & $87.1 \%$ \\
Recall & $85.7 \%$ & $69.7 \%$ & $82.8 \%$ & $88.2 \%$ & $82.4 \%$ \\
Average time (sec.) & 22.9 & 25.2 & 33.55 & 5.15 & 11.9 \\
\hline
\end{tabular}

\section{Experimental Results}

\subsection{Setting of Experiments}

Wo extracted articles related to the release of new products from a onc-year newspaper corpus written in Japanese ${ }^{3}$. One-hundred articles were randomly selected from 362 relevant articles. 'The template we used consisted of five slots: company name, product name, release date, announce date, and price. We also filled one template for each article. After analyzing sentences, case frames wore converted into atomic formulac representing semantic representations as described in Section 2 and 3. All the semantic representations were given to the learner as background knowledge, and the filled templates were given as positive examples. To speed-11p the learning process, we selected predicate names that are relevant to the words in the templates as the target predicates to be used by the IlP system, and we also restricted the number of literals in the body of hypotheses to one.

precision and recall, the standard metrics for IIE tasks, are counted by using the remove-oneout cross validation on the examples for each item. We used a VArStation with the Pentium II Xeon (450 MII\%) for this experiment.

\subsection{Results}

Table 4 shows the results of our experiment. In the experiment of learning from semantic representations, including errors in casc-role selection and semantic category selection, precision was

\footnotetext{
${ }^{3}$ We nsed articles from the Mainiclii Newspajers of 1994 with permission.
}

very high. 'The precision of the learned rules for price was low because the semantic category name automatically given to the price expressions in the data were not quite appropriate. For the five items, $67-82 \%$ recall was achicved. With the background knowledge having semantic representations corrected by hand, precision was very high and $70-88 \%$ recall was achieved. The precision of price was markedly improved.

It is important that the extraction of five different pieces of information showed good tesults. 'This indicates that the IIJ' system RIT $3^{+}$ has a high potential in Il' tasks.

\section{Related Work}

Previous researches on gencrating $\mathrm{IF}$ rules from texts with templates include AutoSlogTS (Riloff,1996), CRYSTAL (Soderland ol al., 1995), PAIKA (Kim el al., 1995), LIEP' (Huffman, 1996) and RAPIER (Califf and Mooncy, 1997). In our approach, we use the typeoriented IJ/' system $\mathrm{RHH}^{+}$, which is independent of natural language analysis. 'This point differentiates our approach from the others. learning scmantic-level IE rules using an. II/P system from semantic representations is also a new challenge in IIE studies.

Sasaki (Sasaki and Haruno, 1997) applied $\mathrm{RHB}^{+}$to the extraction of the number of deaths and injuries from twenty five articles. That experiment was sufficient to assess the porformance of the learner, but not to evaluate its feasibility in IE tasks. 


\section{Conclusions and Remarks}

This paper described a use of semantic representations for generating information extraction rules by applying a type-oriented II.P system. Experiments were conducted on the data generated from 100 news articles in the domain of new product release. The results showed very high precision, recall of $67-82 \%$ without data correction and $70-88 \%$ recall with correct semantic representations. The extraction of five different pieces of information showed good results. This indicates that our learner $\mathrm{RHB}^{+}$has a high potential in IE tasks.

\section{References}

H. Aït-Kaci and R. Nasr, LOGIN: A logic programming language with builti-in inheritance, Journal of Logic Programming, 3, pp.185$215,1986$.

H. Aït-Kaci, B. Dumant, R. Meyer, A. Podelski, and P. Van Roy, The Wild Life Handbook, 1994.

M. E. Califf and R. J. Mooney, Relational Jearning of Pattern-Match Rules for Information Extraction, Proc. of ACL-97 Workshop in Natural Language Learning, 1997.

S. B. Huffman, Learning Information Extraction Patterns from Examples, Statistical and Symbolic Approaches to Learning for Natural Language Processing, pp.246-260, 1996.

S. Ikehara, M. Miyazaki, and A. Yokoo, Classification of language knowledge for meaning analysis in machine translations, Transactions of Information Processing Society of Japan, Vol.34, pp.1692-1704, 1993. (in Japanese)

S. Ikehara, S. Shirai, K. Ogura, A. Yokoo, H. Nakaiwa and T. Kawaoka, ALT-J/E: A Japanese to English Machine Translation System for Communication with Translation, Proc. of The 13th IFIP World Computer Congress, pp.80-85, 1994.

S. Ikehara, M. Miyazaki, S. Shirai, A. Yokoo, H. Nakaiwa, K. Ogura, Y. Oyama and Y. Hayashi (eds.), The Semantic Attribute System, Goi-Taikei - A Japanese Lexicon, Vol.1, Iwanami Publishing, 1997. (in Japanese)

S. Ikehara, M. Miyazaki, S. Shirai, A. Yokoo, H. Nakaiwa, K. Ogura, Y. Oyama and Y. Hayashi (eds.), The Valency Dictionary,
Goi-Taikei - A Japanese Lexicon, Vol.5, Iwanami Publishing, 1997. (in Japancse)

J.-T. Kim and D. I. Moldovan, Acquisition of Linguistic Patterns for Knowledge-Based Information Extraction, IEEE Transaction on Knowledge and Data Engineering (IEEE TKDE), Vol.7, No.5, pp.713-724, 1995.

S. Kurohashi and Y. Sakai, Semantic Analysis of Japanese Noun Phrases: A New Approach to Dictionary-Based Understanding The $37 t h$ Annual Meeting of the Association for Computational Linguistics (ACL-99), pp.481-488, 1999.

W. Lehnert, C. Cardie, D. Fisher, J. McCarthy, E. Riloff and S. Soderland, University of Massachusetts: MUC-4 Test Results and Analysis, Proc. of The Fourth Message Understanding Conference (MUC-4), pp.151-158, 1992.

J. Lloyd, Foundations of Logic Programming, Springer, 1987.

S. Muggleton, Inductive logic programming, New Generation Computing, Vol.8, No.4, pp.295-318, 1991.

F. Riloff, Automatically Generating Extraction Pattern from Untagged Text, Proc.of American Association for Artificial Intelligence (AAAI-96), pp.1044-1049, 1996.

Y. Sasaki and M. Haruno, RHB ${ }^{+}$: A TypeOriented ILP System I Jearning from Positive Data, Proc. of The 14th International Joint Conference on Artificial Intelligence (IJCAI97), pp.894-899, 1997.

S. Soderland, D. Fisher, J. Aseltine, W. Lenert, CRYSTAL: Inducing a Conceptual Dictionary, Proc. of The 13th International Joint Conference on Artificial Intelligence (IJCAI95), pp.1314-1319, 1995.

J. M. Zelle and R. J. Mooney, J. B. Konvisser, Combining Top-down and Bottom-up Methods in Inductive Logic Programming, Proc of The 11th International Conference on $\mathrm{Ma}$ chine Learning (ML-94), pp.343-351, 1994. 